This is the accepted manuscript made available via CHORUS. The article has been published as:

\title{
Transverse collective flow and midrapidity emission of isotopically identified light charged particles
}

Z. Kohley, L. W. May, S. Wuenschel, M. Colonna, M. Di Toro, M. Zielinska-Pfabe, K. Hagel,

R. Tripathi, A. Bonasera, G. A. Souliotis, D. V. Shetty, S. Galanopoulos, M. Mehlman, W. B.

Smith, S. N. Soisson, B. C. Stein, and S. J. Yennello

Phys. Rev. C 83, 044601 - Published 1 April 2011

DOI: 10.1103/PhysRevC.83.044601 


\title{
Transverse Collective Flow and Mid-Rapidity Emission of Isotopically Identified Light Charged Particles
}

\author{
Z. Kohley ${ }^{1,2, *}$ L. W. May, ${ }^{1,2}$ S. Wuenschel,${ }^{1,2}$ M. Colonna, ${ }^{3}$ M. Di Toro,${ }^{3,4}$ M. Zielinska-Pfabe, ${ }^{5}$ \\ K. Hagel,${ }^{2}$ R. Tripathi, ${ }^{2}$ A. Bonasera, ${ }^{2,3}$ G. A. Souliotis, ${ }^{6,2}$ D. V. Shetty, ${ }^{2,} \dagger$ S. Galanopoulos ${ }^{2}$ \\ M. Mehlman, ${ }^{7,2}$ W. B. Smith, ${ }^{2}$ S. N. Soisson, ${ }^{1,2}$ B. C. Stein, ${ }^{1,2}$ and S. J. Yennello ${ }^{1,2}$ \\ ${ }^{1}$ Chemistry Department, Texas AEM University, College Station, Texas 77843, USA \\ ${ }^{2}$ Cyclotron Institute, Texas A\&M University, College Station, Texas 77843, USA \\ ${ }^{3}$ Laboratori Nazionali del Sud, INFN, I-95123 Catania, Italy \\ ${ }^{4}$ Physics and Astronomy Department, University of Catania, Italy \\ ${ }^{5}$ Smith College, Northampton, MA, USA \\ ${ }^{6}$ Laboratory of Physical Chemistry, Department of Chemistry, \\ National and Kapodistrian University of Athens, Athens 15771, Greece \\ ${ }^{7}$ Physics and Astronomy Department, Texas A\&M University, College Station, Texas 77843, USA
}

The transverse flow and relative mid-rapidity yield of isotopically identified light charged particles (LCPs) has been examined for the $35 \mathrm{MeV} /$ nucleon ${ }^{70} \mathrm{Zn}+{ }^{70} \mathrm{Zn},{ }^{64} \mathrm{Zn}+{ }^{64} \mathrm{Zn}$, and ${ }^{64} \mathrm{Ni}+{ }^{64} \mathrm{Ni}$ systems. A large enhancement of the mid-rapidity yield of the LCPs was observed relative to the yield near the projectile rapidity. In particular, this enhancement was increased for the more neutron-rich LCPs demonstrating a preference for the production of neutron-rich fragments in the mid-rapidity region. Additionally, the transverse flow of the LCPs was extracted which provides insight into the average movement of the particles in the mid-rapidity region. Isotopic and isobaric effects were observed in the transverse flow of the fragments. In both cases, the transverse flow was shown to decrease with an increasing neutron content in the fragments. A clear inverse relationship between the transverse flow and relative mid-rapidity yield is shown. The increased relative mid-rapidity emission produces a decreased transverse flow. The Stochastic Mean-Field model was used for comparison to the experimental data. The results showed that the model was able to reproduce the general isotopic and isobaric trends for the mid-rapidity emission and transverse flow. The sensitivity of these observables to the density dependence of the symmetry energy was explored. The results indicate that the transverse flow and mid-rapidity emission of the LCPs are sensitive to the denisty dependence of the symmetry energy.

PACS numbers: 25.75.Ld, 25.70.Pq, 25.70.-z,21.65.Ef

\section{INTRODUCTION}

Improving our understanding of the nuclear Equation of State (EoS) is an important goal for the field of nuclear science. Currently, the EoS for symmetric nuclear matter is thought to be relatively constrained, while predictions for the EoS of asymmetric nuclear matter can still vary widely [1-3]. Recent experimental results are being used to apply constraints to the asymmetric part of the EoS, or density dependence of the symmetry energy $\left(\mathrm{E}_{\text {sym }}(\rho)\right)[3-6]$. Constraining the density dependence of the symmetry energy is essential for understanding the fundamental nucleon-nucleon interaction and has important astrophysical implications [3, 5, 7-13].

Heavy-ion collisions (HICs) provide a unique opportunity to examine the nuclear EoS since nuclear matter is produced at temperatures, densities, and neutronto-proton $(\mathrm{N} / \mathrm{Z})$ ratios away from that of ground state

*Electronic address: zkohley@comp.tamu.edu; Current Address: Physics Division, Oak Ridge National Laboratory, Oak Ridge, TN 37831, USA

†Physics Department, Western Michigan University, Kalamazoo, MI 49008, USA nuclei. In the examination of peripheral and semiperipheral intermediate energy heavy-ion collisions an important source of particle production has been found to originate from a mid-rapidity, or neck, region between the quasi-projectile, QP, and quasi-target, QT [14-19]. Experimental results have demonstrated an increased neutron-to-proton ratio in this mid-rapidity region in comparison to the quasi-projectile source through the examination of isotopically resolved fragments as well as the detection of free neutrons [18-26]. For example, Lukasik et al. showed that $65-70 \%$ of the total ${ }^{3} \mathrm{H}$ production can be attributed to the mid-rapidity region [18]. Furthermore, it has been suggested that the neck-like structure represents a low-density region of nuclear matter between the higher density QP and QT [27, 28]. Thus, the neck region can provide an opportunity to examine dilute neutron-rich nuclear matter. The study of this low-density asymmetric nuclear matter should provide sensitivity to the nuclear EoS. Theoretical models have shown that the isospin content and production of intermediate mass fragments (IMFs) in the neck region could be used to probe the nuclear EoS [17, 29-31].

The transverse flow is closely connected to the midrapidity emission properties and describes the average movement of the particles in the mid-rapidity region. 
While transverse flow measurements have been very important in helping elucidate the EoS for symmetric nuclear matter $[32,33]$, they have been consistently discussed as a probe to examine the density dependence of the symmetry energy [3,34-37]. Pak et al. demonstrated that both the the transverse collective flow for $\mathrm{Z}=1-3$ particles and the balance energy increased with an increasing neutron-to-proton ratio of the system, $(\mathrm{N} / \mathrm{Z})_{\text {sys }}[38,39]$. This was the first evidence that the collective flow was sensitive to the isospin concentration of the colliding system. The isospin dependence of the transverse flow and balance energy were attributed to the isospin-dependent potential and in-medium nucleon-nucleon cross sections through comparisons with a BUU and quantum molecular dynamics (QMD) model [40, 41]. Scalone et al. used a Boltzmann-Nordheim-Vlasov (BNV) simulation to demonstrate that the isospin dependence observed by Pak et al. was sensitive to the density dependence of the symmetry energy [42].

Differences between the free neutron and free proton transverse collective flows have been predicted to be strongly sensitive to $\mathrm{E}_{\text {sym }}(\rho)$ [43-45]. However, obtaining accurate energy and angular measurements of free neutrons, along with charged particles, is a difficult task. Currently, experimental data from the FOPI/LAND detectors are being used to extract neutron and proton collective flows in order to examine the sensitivity to the density dependence of the symmetry energy $[46,47]$. The results will be used to plan a dedicated experiment in an attempt to apply high density constraints to the symmetry energy.

Scalone et al. showed that a comparison of the flow parameter from ${ }^{3} \mathrm{H}$ and ${ }^{3} \mathrm{He}$ light clusters would exhibit a similar dependence on $\mathrm{E}_{\text {sym }}(\rho)$ as the neutron and proton flows [42]. The simulations showed that for a stiff symmetry energy parameterization the ${ }^{3} \mathrm{He}$ clusters should have an estimated $20 \%$ larger flow than the ${ }^{3} \mathrm{H}$ clusters in midperipheral collisions from a $55 \mathrm{MeV} /$ nucleon ${ }^{58} \mathrm{Fe}+{ }^{58} \mathrm{Fe}$ reaction system [42]. In the case of a soft parameterization, the difference in the flow parameter between the ${ }^{3} \mathrm{H}$ and ${ }^{3} \mathrm{He}$ clusters disappeared [35, 42]. Thus, by measuring the flow parameter of the ${ }^{3} \mathrm{H}$ and ${ }^{3} \mathrm{He}$ clusters one should be able to gain insight into the density dependence of the symmetry energy. Recently, Yong et al., using a BUU calculation, have demonstrated that the ${ }^{3} \mathrm{H}$ and ${ }^{3} \mathrm{He}$ flow, from a $400 \mathrm{MeV} /$ nucleon reaction, could be used to probe the density dependence of the symmetry energy at supra-saturation densities [48].

In the this paper, the relative mid-rapidity emission and the transverse flow of isotopically identified light charged particles has been investigated for the 35 $\mathrm{MeV} /$ nucleon ${ }^{70} \mathrm{Zn}+{ }^{70} \mathrm{Zn}$, ${ }^{64} \mathrm{Zn}+{ }^{64} \mathrm{Zn}$, and ${ }^{64} \mathrm{Ni}+{ }^{64} \mathrm{Ni}$ systems. The experimental details are provided in Section II, along with a brief description of the Stochastic Mean-Field (SMF) model $[49,50]$. The experimental and simulated mid-rapidity yields and transverse flow results are presented in Section III. Lastly, the conclusions and acknowledgements are provided in Sections IV and V, respectively.

\section{EXPERIMENTAL AND THEORETICAL DETAILS}

\section{A. Detector Set-Up}

The K500 Superconducting Cyclotron at the Texas A\&M University Cyclotron Institute was used to produce beams of ${ }^{70} \mathrm{Zn},{ }^{64} \mathrm{Zn}$, and ${ }^{64} \mathrm{Ni}$ at $35 \mathrm{MeV} /$ nucleon which were collided with ${ }^{70} \mathrm{Zn}(95 \%),{ }^{64} \mathrm{Zn}(99.8 \%)$, and ${ }^{64} \mathrm{Ni}$ $(98.0 \%)$ self-supporting targets, respectively. The reaction products were measured using the $4 \pi$ NIMROD-ISiS array (Neutron Ion Multi-detector for Reaction Oriented Dynamics with the Indiana Silicon Sphere) [51]. The entire charged particle array is housed inside the Texas A\&M Neutron Ball [52], which provides an average neutron multiplicity.

The charged particle array consists of 14 concentric rings, labeled rings $2-15$, covering from $3.6^{\circ}$ to $167.0^{\circ}$ in lab. Rings $2-9$, ranging from $3.6^{\circ}$ to $45.0^{\circ}$, have the same geometry as the INDRA detector [53] and rings 10-15 are of the ISiS geometry [54]. Rings 2-9 each consist of 10 single telescope modules and 2 super telescope modules. A single telescope module contains a $150 \mu \mathrm{m}$ or $300 \mu \mathrm{m}$ silicon detector placed in front of a thallium doped cesium iodide crystal, $\mathrm{CsI}(\mathrm{Tl})$. The super telescopes have both a $150 \mu \mathrm{m}$ and $500 \mu \mathrm{m}$ Si placed in front of the $\mathrm{CsI}(\mathrm{Tl})$ crystal. Rings 10 and 11 each have 18 single telescope modules with $300 \mu \mathrm{m}$ Si-CsI(Tl) detectors. Rings 12-15 each contain 18 single telescope detectors, with $500 \mu \mathrm{m}$ thick silicon.

Three methods of particle identification are available in the NIMROD-ISiS array. In rings $2-11$ pulse shape analysis of the $\mathrm{CsI}(\mathrm{Tl})-\mathrm{PMT}$ (thallium doped cesium-iodide with a photomultiplier tube) signals provided clear separation of neutron/gamma, ${ }^{1} \mathrm{H},{ }^{2} \mathrm{H},{ }^{3} \mathrm{H},{ }^{3} \mathrm{He},{ }^{4} \mathrm{He}$, and ${ }^{6} \mathrm{He}$ particles. Isotopic resolution of heavier mass fragments was achieved through $\Delta \mathrm{E}-\mathrm{E}$ measurements from the Si-CsI (single telescope) and $\mathrm{Si}-\mathrm{Si}$ (super telescope) modules. In the forward angle rings, isotopic resolution of $1 \leq Z \leq 17$ particles and elemental resolution up to the charge of the beam was obtained through the Si-CsI and Si-Si detector modules. Detector thresholds limited the isotopic resolution to $1 \leq Z \leq 2$ particles for the backward angles. A linearization procedure was utilized to complete the particle identification $[51,55,56]$.

The relationship derived by Tasson-Got [57] was used to relate the light output from the CsI to the particle energy. Proton $(30$ and $55 \mathrm{MeV}),{ }^{2} \mathrm{H}(60 \mathrm{MeV})$, and ${ }^{4} \mathrm{He}(100 \mathrm{MeV})$ calibrations beams were used in order help constrain the parameters of the CsI calibration. The punch-through energies of the identified fragments, along with a ${ }^{228} \mathrm{Th}$ source and a $500 \mathrm{MeV}{ }^{20} \mathrm{Ne}$ calibration beam, were used to constrain the silicon detector calibrations. The resulting energy spectra were compared to previous NIMROD data sets for $35 \mathrm{MeV} /$ nucleon systems 
of similar size and showed excellent agreement [58, 59].

\section{B. Event Selection and Reaction Plane Determination}

In examining the mid-rapidity emission and transverse collective flow it is important to estimate the centrality of the collisions since the strongest signatures are observed in mid-peripheral collisions $[18,35,38,60]$. The impact parameter, for the experimental data, was estimated using minimum bias distributions of the neutron multiplicity plotted against the charged particle multiplicity for each system. Three bins were created from the 2-D distributions such that each bin would represent $\mathrm{a} b / \mathrm{b}_{\max }$, or $\mathrm{b}_{\text {red }}$, width of 0.33 if one assumes a corresponding triangular impact parameter distribution. A simulation with the Constrained Molecular Dynamics Model [61], filtered for experimental acceptance, confirmed this method of impact parameter selection. Thus, the bin representing the events with the highest neutron and charged particle multiplicities should represent the most central collisions, while the low-multiplicity events should represent the most peripheral collisions. In the following, the transverse flow is examined for the mid-peripheral collisions, which should represent $\mathrm{b}_{\text {red }} \cong 0.33-0.66$. In order to ensure that a well-characterized system was considered an event criterion was imposed such that the total detected charge for an event must be greater than $40 \%$ of the total charge in the colliding system.

The azimuthal correlation method [62] was used for the reaction plane reconstruction from the transverse momentum of the fragments of each event. The azimuthal correlation method does not differentiate between the forward, quasi-projectile, and the backward, quasi-target, sides of the flow. Therefore, the forward flow side of the reaction plane was determined using the transverse momentum analysis method [63]. The particle of interest (POI) was removed from the calculation of the reaction plane in order to avoid autocorrelations [62-64]. Thus, the reaction plane was calculated for each particle in an event rather than on an event by event basis. A velocity boost was applied to each particle used in the calculation of the reaction plane to account for momentum conservation $[62,65]$.

The method described in Ref. [62] was used to estimate the accuracy of the reaction plane resolution. Each event was randomly divided into two sub-events and the reaction plane was calculated for each of the sub-events. The difference in the reaction-plane angle between the sub-events, $\Delta \phi_{12}$, was then calculated. The width of the $\Delta \phi_{12}$ distribution can be related to the standard deviation between the reconstructed and true reaction plane, which is a representation of the reaction plane resolution [62]. The standard deviation between the true and reconstructed reaction plane was estimated to be $\sim 27^{\circ}$, which demonstrates an improved accuracy in comparison to previous experiments [62].

\section{Stochastic Mean-Field Model}

The mid-rapidity emission and transverse flow of the LCPs was investigated within the framework of the Stochastic Mean-Field (SMF) model [49, 50]. The SMF model uses the test-particle method to solve the Boltzmann-Langevin transport equation [66]. A momentum dependent potential was used in the simulation and the density dependence of the symmetry energy was varied $[49,50]$. In the following, the stiff SMF and soft SMF results will refer to the results of the SMF model where the potential produces a stiff and soft, respectively, density dependence of the symmetry energy. The simulation was stopped at $120 \mathrm{fm} / \mathrm{c}$ and a phase-space coalescence was applied to identify the fragments [67]. Thus, theoretical simulation provides information about the initial fragment distribution.

\section{RESULTS AND DISCUSSION}

In the following section, both the LCP yield and $\left\langle P_{x} / A\right\rangle$ are examined as a function of the reduced rapidity, which is defined as

$$
Y_{r}=\frac{Y_{c m}}{Y_{c m, p r o j}}
$$

where $\mathrm{Y}_{c m}$ is the center-of-mass rapidity of the LCP and $\mathrm{Y}_{c m, p r o j}$ is the center-of-mass rapidity of the projectile [68]. In mass symmetric systems, this conveniently scales the rapidity such that $Y_{r}=1(-1)$ is equal to the projectile (target) rapidity. Therefore, the mid-rapidity region is easily defined around $\mathrm{Y}_{r}=0$.

\section{A. Mid-Rapidity Emission}

In Figure 1 the yields of the different isotopically identified LCPs (solid black circles) are shown as a function of the reduced rapidity from the experimental data (the SMF results will be discussed below). The observed asymmetry in the distributions are due to the decreased efficiency for detection of the LCPs at the backward lab angles due to the lower lab-frame energy of the fragments. Therefore, we will focus on the forward rapidity region $\left(Y_{r}>0\right)$ where the detector capabilities are much better. The results demonstrate a clear preference for emission around the mid-rapidity region $\left(\mathrm{Y}_{r}=0\right)$ for all LCPs, in comparison to where one would expect to observe the decay of the quasi-projectile (QP), near $\mathrm{Y}_{r}=1$.

To further explore the enhanced mid-rapidity emission the relative yield of the LCPs was calculated as,

$$
R_{Y i e l d}^{M i d}=\frac{Y_{i e l d^{M i d-R a p i d i t y ~}}}{Y_{\text {ield }}^{Q P} / 2}
$$

where the mid-rapidity yield (Yield ${ }^{\text {Mid-Rapidity }}$ ) is defined as the yield from $0.0 \leq \mathrm{Y}_{r} \leq 0.5$ and the QP yield 


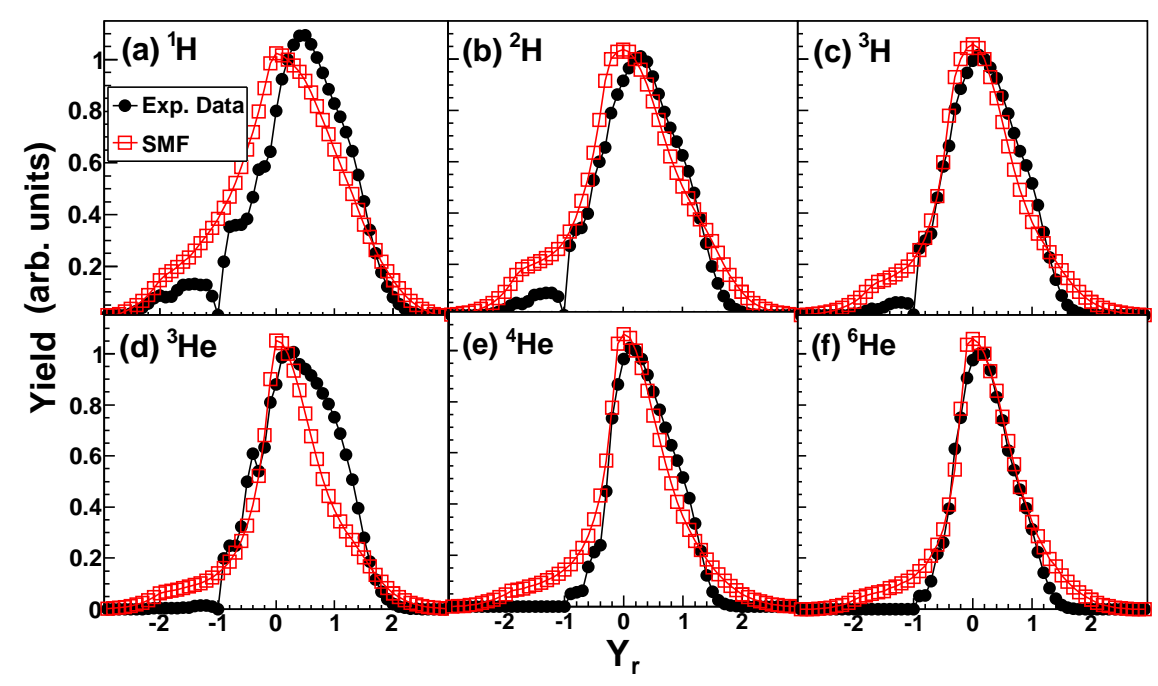

FIG. 1: (Color online) Reduced rapidity $\left(\mathrm{Y}_{r}\right.$ ) distribution for (a) ${ }^{1} \mathrm{H}$, (b) ${ }^{2} \mathrm{H}$, (c) ${ }^{3} \mathrm{H}$, (d) ${ }^{3} \mathrm{He}$, (e) ${ }^{4} \mathrm{He}$, and (f) ${ }^{6} \mathrm{He}$ fragments from the $35 \mathrm{MeV} /$ nucleon ${ }^{64} \mathrm{Ni}+{ }^{64} \mathrm{Ni}$ reaction. The experimental data are shown as the solid black circles and the stiff SMF calculation is shown as the open red squares. The detector thresholds have been applied to the SMF simulation. Each distribution has been normalized such that the yield at $\mathrm{Y}_{r}=0.0$ equals unity.

(Yield ${ }^{Q P}$ ) is defined as the yield from $0.5 \leq \mathrm{Y}_{r} \leq 1.5$. Notice that Yield ${ }^{Q P}$ was scaled by a factor of 2 in Eq. 2 since a larger range was used in defining the QP yield. It is important to recognize that these definitions for the mid-rapidity and QP yield are very simple estimations, however they should provide some insight into the relative production of the LCPs.

The $\mathrm{R}_{Y \text { ield }}^{\text {Mid }}$ values for the LCPs are presented in Figure 2 as a function of the charge times mass (ZA) for the 3 reaction systems. A distinct trend is observed demonstrating an increased mid-rapidity yield for the more neutron-rich LCPs. For the $\mathrm{Z}=1$ isotopes, the largest value of $\mathrm{R}_{Y \text { ield }}^{\text {Mid }}$ is shown for ${ }^{3} \mathrm{H}(\mathrm{N} / \mathrm{Z}=2.0)$ followed by the ${ }^{2} \mathrm{H}(\mathrm{N} / \mathrm{Z}=1.0)$ and lastly, ${ }^{1} \mathrm{H}(\mathrm{N} / \mathrm{Z}=0.0)$. The same trend is also present for the $Z=2$ isotopes with the ${ }^{6} \mathrm{He}$ $(\mathrm{N} / \mathrm{Z}=2.0)$ showing the largest mid-rapidity enhancement and the ${ }^{3} \mathrm{He}(\mathrm{N} / \mathrm{Z}=0.5)$ showing the least. Additionally, an isobaric comparison between ${ }^{3} \mathrm{H}$ and ${ }^{3} \mathrm{He}$, shows the same trend with a larger fraction of ${ }^{3} \mathrm{H}$ being produced in the mid-rapidity region than ${ }^{3} \mathrm{He}$ fragments. While the actual N/Z of the mid-rapidity region relative to the QP region cannot be determined without free neutron detection [20], these results demonstrate a strong preference for the emission of neutron-rich LCPs into the mid-rapidity region. These results are in agreement with previous works showing an increased production of neutron-rich fragments in the mid-rapidity region [17$26]$.

In comparing the $\mathrm{R}_{Y \text { ield }}^{\mathrm{Mid}}$ results between systems relatively small differences are observed. Particularly, for the $\mathrm{Z}=1$ isotopes there is not a large difference in the midrapidity yield between the different reaction systems, as shown in Figure 2. The most significant system effects are

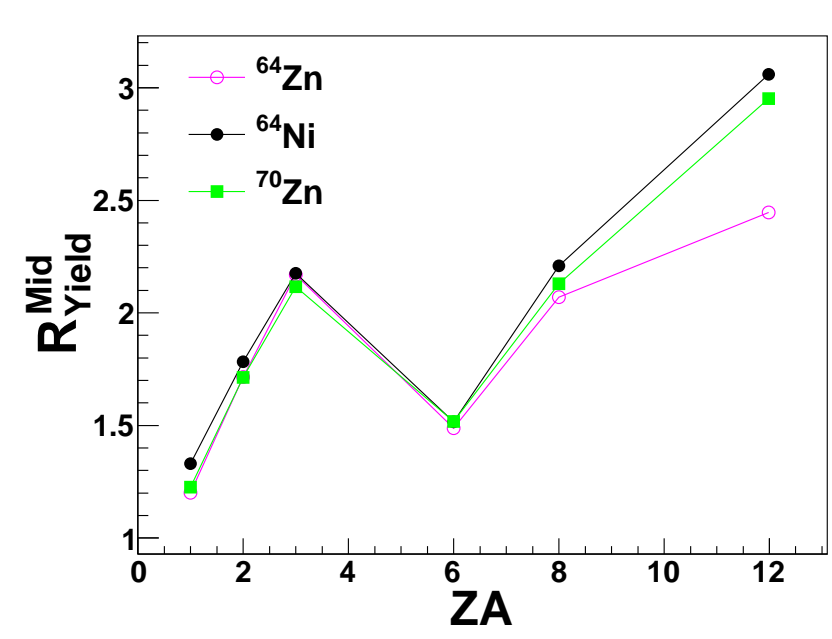

FIG. 2: (Color online) $\mathrm{R}_{Y \text { ield }}^{\text {Mid }}$ values are shown as a function of the charge times mass ( $\mathrm{ZA})$ for ${ }^{1} \mathrm{H}(\mathrm{ZA}=1),{ }^{2} \mathrm{H}(\mathrm{ZA}=2)$, ${ }^{3} \mathrm{H}(\mathrm{ZA}=3),{ }^{3} \mathrm{He}(\mathrm{ZA}=6),{ }^{4} \mathrm{He}(\mathrm{ZA}=8)$, and ${ }^{6} \mathrm{He}(\mathrm{ZA}=12)$ particles.

observed for the ${ }^{6} \mathrm{He}$ fragments where a large difference in the mid-rapidity yield is observed for the ${ }^{64} \mathrm{Zn}+{ }^{64} \mathrm{Zn}$ system in comparison to the ${ }^{64} \mathrm{Ni}+{ }^{64} \mathrm{Ni}$ and ${ }^{70} \mathrm{Zn}+{ }^{70} \mathrm{Zn}$ systems. The relative mid-rapidity ${ }^{6} \mathrm{He}$ yield is suppressed in the least neutron-rich, ${ }^{64} \mathrm{Zn}$, system, while the more neutron-rich systems show a strong enhancement of the mid-rapidity ${ }^{6} \mathrm{He}$ emission.

The rapidity distributions for the LCPs calculated by the SMF model are compared to the experimental distributions in Figure 1. The experimental detector thresholds have been applied to the SMF model calculation. 


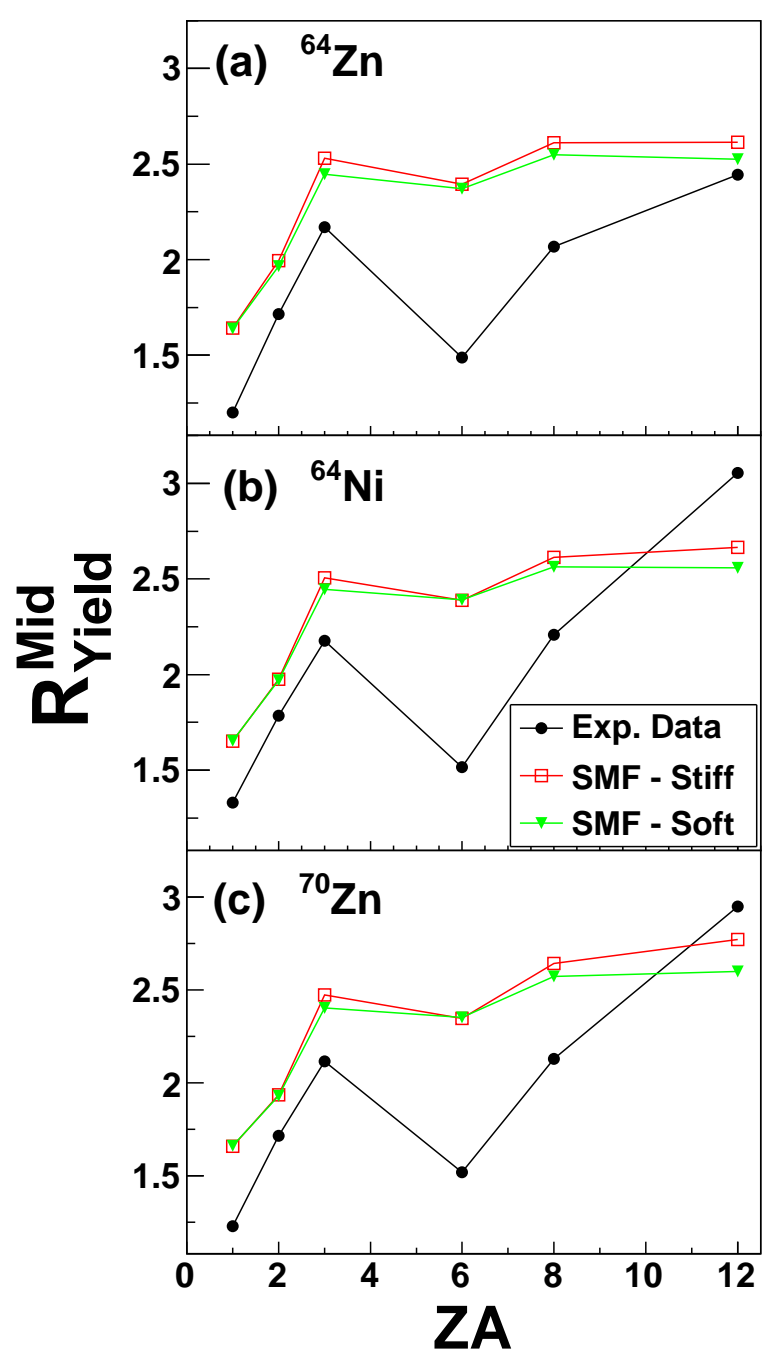

FIG. 3: (Color online) Comparison of the $\mathrm{R}_{\text {Yield }}^{\text {Mid }}$ values from the experimental data (solid black circles) with the stiff (open red squares) and soft (solid green triangles) SMF calculation. The results from the 3 reaction systems are shown (panels $\mathrm{a}-\mathrm{c})$ and are plotted as a function of the charge times mass (ZA) of the LCPs.

The comparison shows that the SMF model is able to reasonably reproduce the experimental rapidity distributions. Both the simulation and experiment demonstrate a strong preference for particle emission around the midrapidity region $\left(\mathrm{Y}_{r}=0.0\right)$. The largest discrepancies are observed for the proton and ${ }^{3} \mathrm{He}$ particles where the experimental distribution tends to have a increased forward rapidity yield relative to the SMF model. This difference could be attributed to the statistical decay of the QP at later stages of the reaction, which is not present in the SMF model, and has been shown to produce a significant fraction of the proton and ${ }^{3} \mathrm{He}$ yield $[18,19]$.

The preferential emission of neutron-rich LCPs into the mid-rapidity region can be examined with the SMF model by examining the $\mathrm{R}_{Y \text { ield }}^{\mathrm{Mid}}$ values, as shown in Fig- ure 3. In comparison to the experimental data the SMF calculation, in general, overestimates the mid-rapidity enhancement of the LCPs. As mentioned, this may be due to the lack of QP decay which would lower the $\mathrm{R}_{Y i e l d}^{\mathrm{Mid}}$ values. However, the SMF calculations do show an increasing mid-rapidity yield for the more neutronrich LCPs. In examining the isotopic trends, the SMF model shows a very similar trend for the $\mathrm{Z}=1$ isotopes as the experimental data. A strong increase in the ${ }^{3} \mathrm{H}$ mid-rapidity yield is observed relative to the proton yield. For the $\mathrm{Z}=2$ isotopes, the SMF model shows an increased $\mathrm{R}_{Y i e l d}^{\mathrm{Mid}}$ with increasing neutron content of the fragments; however, the magnitude of the increase is smaller than that observed in the experimental data. Similarly, the isobaric comparison from the SMF calculation shows the same trend as the experimental data, with an increased mid-rapidity yield of the neutron-rich ${ }^{3} \mathrm{H}$ fragments relative to the ${ }^{3} \mathrm{He}$ fragments, yet the magnitude of the difference is much smaller than that observed in the experiment. Even though there are clear differences between the theory and experiment, the results demonstrate the ability of the SMF model to correctly predict the enhanced particle production (Figure 1) and neutron enrichment (Figure 3) of the mid-rapidity region. Also, the impact of the density dependence of the symmetry energy can be seen in Figure 3, where an increased mid-rapidity yield for the neutron-rich ${ }^{3} \mathrm{H}$ and ${ }^{6} \mathrm{He}$ isotopes is observed with a stiff density dependence of the symmetry energy.

\section{B. Transverse Flow}

The transverse flow is often quantified as the slope of the average in-plane momentum, $\left\langle P_{x}\right\rangle$, over the midrapidity region. In Figure 4, the average in-plane momentum per nucleon is plotted as a function of the reduced rapidity $\left(\mathrm{Y}_{r}\right)$, for the different isotopically identified LCPs. The solid line, shown in each panel, represents a linear fit over the region $-0.35 \leq \mathrm{Y}_{r} \leq 0.35$. The extracted slope of the linear fit represents the transverse flow of the LCPs and is referred to as the flow parameter. This range was chosen since the smooth curvature of the $\left\langle P_{x} / A\right\rangle$ changes near $\mathrm{Y}_{r}=-0.35$ due to the backward angle detector thresholds. The error in the flow parameter was calculated from the error in the linear fit. Systematic errors associated with the fit range were estimated to be at most +1 and $-0.4(\mathrm{MeV} / \mathrm{c}) / \mathrm{A}$, by varying the fit range between $-0.4 \leq \mathrm{Y}_{r} \leq 0.4$ and $-0.15 \leq \mathrm{Y}_{r} \leq$ 0.15 . Systematic error from the SMF model was negligible at +0.3 and $-0.2(\mathrm{MeV} / \mathrm{c}) / \mathrm{A}$. Unless noted, only the fit error is presented in the results below.

The transverse flow of the $\mathrm{Z}=1$ and $\mathrm{Z}=2$ particles is presented first to examine the dependence of the flow on the mass, charge, and isospin content of the system. Additionally, this provides an opportunity to compare our results with those of Pak et al. [38] in a relatively straightforward manner. In Figure 5 the $\mathrm{Z}=1$ and $\mathrm{Z}=2$ transverse flow is shown as a function of the neutron-to-proton ra- 


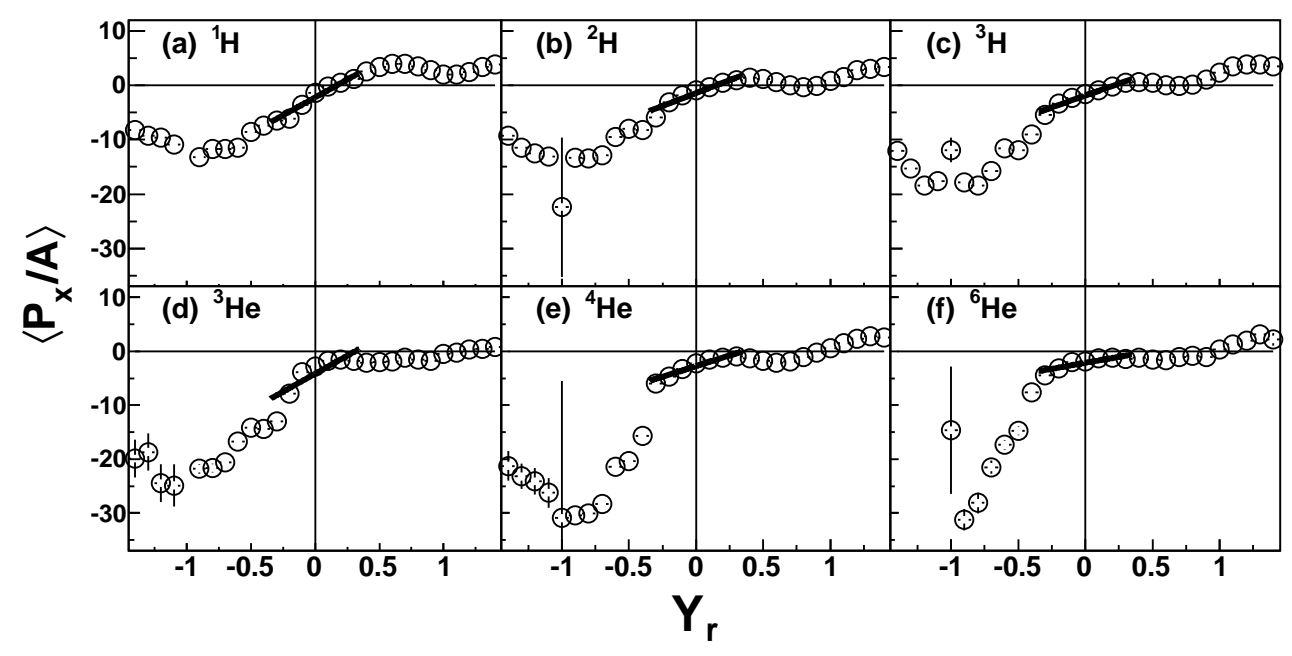

FIG. 4: Average in-plane momentum, $\left\langle P_{x} / A\right\rangle$, as a function of the reduced rapidity for (a) ${ }^{1} \mathrm{H},(\mathrm{b}){ }^{2} \mathrm{H}$, (c) ${ }^{3} \mathrm{H}$, (d) ${ }^{3} \mathrm{He},(\mathrm{e})$ ${ }^{4} \mathrm{He}$, and (f) ${ }^{6} \mathrm{He}$ particles. The results shown are from the mid-peripheral collisions of the ${ }^{64} \mathrm{Ni}+{ }^{64} \mathrm{Ni}$ system. The solid black line represents a linear fit from $-0.35 \leq \mathrm{Y}_{r} \leq 0.35$.

tio of the colliding system, $(\mathrm{N} / \mathrm{Z})_{\text {sys }}$. Focusing on the ${ }^{64} \mathrm{Ni}+{ }^{64} \mathrm{Ni}(\mathrm{N} / \mathrm{Z}=1.28)$ and ${ }^{64} \mathrm{Zn}+{ }^{64} \mathrm{Zn}(\mathrm{N} / \mathrm{Z}=1.13)$ systems, a clear dependence on on the $(\mathrm{N} / \mathrm{Z})_{\text {sys }}$ is present. The results show an increased flow in the more neutronrich system for the $\mathrm{Z}=1$ and $\mathrm{Z}=2$ particles. This is consistent with the previous observation by Pak et al. for $\mathrm{Z}=1$ 3 fragments from systems with the same mass $\left(\mathrm{A}_{\text {sys }}\right)$ [38]. This observation was attributed to the isospin dependence of the mean-field and the nucleon-nucleon (nn) cross-sections [38]. However, based on a recent theoretical work, the observed difference may also be attributed to the increased Coulomb repulsion in the less neutronrich system [69].

The results from the most neutron-rich ${ }^{70} \mathrm{Zn}$ $(\mathrm{N} / \mathrm{Z}=1.33)$ system show a decreased flow in comparison to the ${ }^{64} \mathrm{Ni}(\mathrm{N} / \mathrm{Z}=1.28)$ system. The difference between the $\mathrm{A}_{\text {sys }}=128$ and $\mathrm{A}_{\text {sys }}=140$ systems can be qualitatively understood through the dependence of the transverse flow on the attractive mean-field, repulsive nn-collisions, and Coulomb repulsion. The flow should decrease as a function of $\mathrm{A}_{\text {sys }}$ [70], due to the increased number of nncollisions (which scale with $\mathrm{A}_{\text {sys }}$ ) relative to the attractive mean field (which scales with $\mathrm{A}_{\text {sys }}^{2 / 3}$ ). Additionally, the flow from the ${ }^{70} \mathrm{Zn}$ system should be reduced due to the increased Coulomb force in comparison to the ${ }^{64} \mathrm{Ni}$ system [69].

Lastly, the ${ }^{64} \mathrm{Zn}$ system and the ${ }^{70} \mathrm{Zn}$ system, which have the same Coulomb repulsion, have nearly equal flow. The mass dependence, mentioned above, would imply that the ${ }^{70} \mathrm{Zn}$ system should have a decreased flow. However, the observation that the ${ }^{70} \mathrm{Zn}$ flow is not decreased demonstrates the effect of the isospin dependent mean-field and nn-collision cross section [38]. The results from Figure 5 exhibit the effects of the mass, charge, and isospin dependent components of the transverse flow.

The results of Figure 5 can be expanded upon through

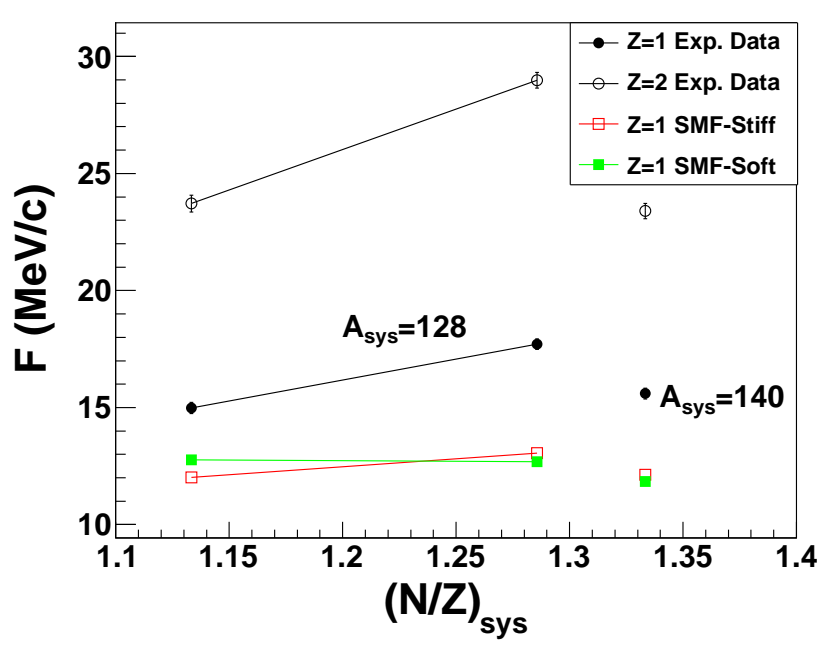

FIG. 5: (Color online) The experimental flow parameters (F) for the $Z=1$ and $Z=2$ fragments are shown as a function of the $\mathrm{N} / \mathrm{Z}$ of the colliding system, $(\mathrm{N} / \mathrm{Z})_{\text {sys }}$, for the mid-peripheral collisions. The Stochastic Mean-Field (SMF) model results are shown for the $\mathrm{Z}=1$ fragments for a stiff and soft $\mathrm{E}_{\text {sym }}(\rho)$. The ${ }^{64} \mathrm{Zn}$ and ${ }^{64} \mathrm{Ni}$ systems, $\mathrm{A}_{\text {sys }}=128$, are connected by a solid line.

examining the flow per nucleon of isotopically identified light charged particles, as shown in Figure 6a. Again, an enhancement in the transverse flow for the ${ }^{64} \mathrm{Ni}$ system is observed in comparison to the ${ }^{64} \mathrm{Zn}$ system demonstrating that the $(\mathrm{N} / \mathrm{Z})_{\text {sys }}$ dependence is also present for the $\mathrm{Z}=1(\mathrm{H})$ and $\mathrm{Z}=2(\mathrm{He})$ isotopes. Additionally, the results from the ${ }^{70} \mathrm{Zn}$ system show, for all isotopes except ${ }^{3} \mathrm{H}$ and ${ }^{3} \mathrm{He}$, a decreased flow in comparison to the $\mathrm{A}_{\text {sys }}=128$ systems, as expected from the $\mathrm{Z}=1$ and $\mathrm{Z}=2$ flow. It is interesting to note, that the observed trend in the flow from the reaction systems, with ${ }^{64} \mathrm{Ni}$ flow $>$ 

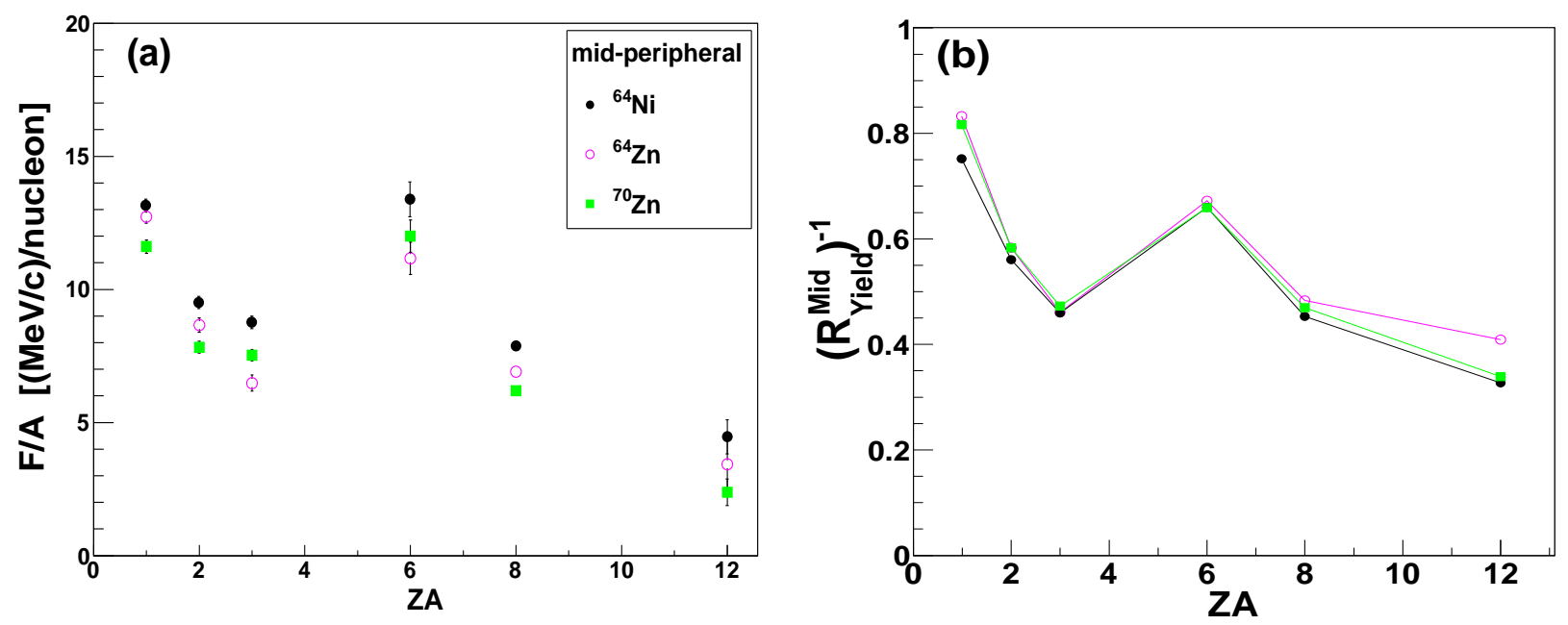

FIG. 6: (Color online) The extracted flow parameters (F) per nucleon (panel a) and inverse $\mathrm{R}_{\text {Yield }}^{\text {Mid }}$ values (panel b) for the ${ }^{1} \mathrm{H},{ }^{2} \mathrm{H},{ }^{3} \mathrm{H},{ }^{3} \mathrm{He},{ }^{4} \mathrm{He}$, and ${ }^{6} \mathrm{He}$ particles are shown as a function of the charge times mass (ZA) of the particle. Results are presented from ${ }^{64} \mathrm{Ni},{ }^{64} \mathrm{Zn}$, and ${ }^{70} \mathrm{Zn}$ systems for mid-peripheral collisions as shown by the legend.

${ }^{64} \mathrm{Zn}$ flow $>{ }^{70} \mathrm{Zn}$ flow, is not present for the ${ }^{3} \mathrm{H}$ and ${ }^{3} \mathrm{He}$ fragments where the flow from the ${ }^{70} \mathrm{Zn}$ system is larger than that from the ${ }^{64} \mathrm{Zn}$ system.

Isotopic and isobaric trends can also be explored from the extracted flow parameters in Figure 6a. A clear isotopic trend is observed, in which the transverse flow per nucleon is decreasing as a function of the mass, or $\mathrm{N} / \mathrm{Z}$, of the isotope. For the $\mathrm{Z}=1$ isotopes, the protons exhibit the largest flow followed by ${ }^{2} \mathrm{H}$ and then ${ }^{3} \mathrm{H}$. Similarly, for the $\mathrm{Z}=2$ isotopes, the ${ }^{3} \mathrm{He}$ fragments have the largest flow followed by ${ }^{4} \mathrm{He}$ and then ${ }^{6} \mathrm{He}$. Thus, the flow is seen to decrease as the neutron content of the isotopes increases. Examination of the transverse flow of the ${ }^{3} \mathrm{H}$ and ${ }^{3} \mathrm{He}$ fragments provides an isobaric comparison. The results, as shown in Figure 6a, demonstrate an enhancement in the ${ }^{3} \mathrm{He}$ flow in comparison to the ${ }^{3} \mathrm{H}$ flow. This, again, demonstrates a decreasing flow with increasing neutron content. Therefore, in comparing fragments with a constant charge (isotopes) or a constant mass (isobars) a consistent trend is observed showing a decreased flow for the more n-rich fragments. This suggests a differential movement of neutrons and protons in the dynamics of the mid-peripheral heavy-ion collisions below the balance energy.

The transverse flow extracted from the SMF model calculations was compared to the experimental results. Even though the simulation was stopped at $120 \mathrm{fm} / \mathrm{c}$, previous theoretical results have shown that the magnitude of the transverse flow should saturate before 100 $\mathrm{fm} / \mathrm{c}$ [71-74]. Also, since the beam energy is below the balance energy for the reaction systems the transverse flow extracted from the SMF model is negative. Therefore, in order to compare the SMF model to the experimental data, in which the flow is positive by convention [62], the sign of the flow from the SMF model was changed to be positive.
The $(\mathrm{N} / \mathrm{Z})_{\text {sys }}$ dependence of the $\mathrm{Z}=1$ flow from the SMF model is compared to the experimental data in Figure 5. The magnitude of the $\mathrm{Z}=1$ flow is underestimated by the SMF model. However, the trend showing an increased flow for the ${ }^{64} \mathrm{Ni}$ system relative to the ${ }^{64} \mathrm{Zn}$ system is reproduced with the stiff symmetry potential. This is not the case for the soft $\mathrm{E}_{\text {sym }}(\rho)$ calculation. This suggests a sensitivity to the low-density region of the symmetry potential, where the soft potential is more repulsive causing the more neutron-rich ${ }^{64} \mathrm{Ni}$ system to have a decreased flow relative to the ${ }^{64} \mathrm{Zn}$ system. It should be noted that other theoretical calculations have shown that, while the difference between the flow of equivalent mass system is sensitive to the symmetry energy, the dominant force is the Coulomb repulsion [69]. The $\mathrm{Z}=2$ results from the SMF model are not shown in Figure 5 because there was no statistical difference in the flow between the three systems.

In Figure 7 the LCP flow from the SMF model is compared to the experimental results. In general the magnitude of the fragment flows from the SMF model are larger than that from the experiment. This may be due to the reaction plane dispersion $[63,75,76]$ present in the experimental analysis. However, the SMF model is able to reproduce the fragment flow trends reasonably well. Except for the proton flow, the isotopic trends show a decreasing flow with an increasing neutron content, as was observed in the experimental data. The SMF model is also able to reproduce the decreased difference in the ${ }^{2} \mathrm{H}$ and ${ }^{3} \mathrm{H}$ flow observed in the the ${ }^{70} \mathrm{Zn}$ experimental data (Figure 7c). The agreement between the fragment flow trends suggests that the SMF model correctly calculates a differential movement of the neutrons and protons in the mean-field. Thus, in both the experiment and theory a decreased flow is observed with increasing neutron content of the fragments. 


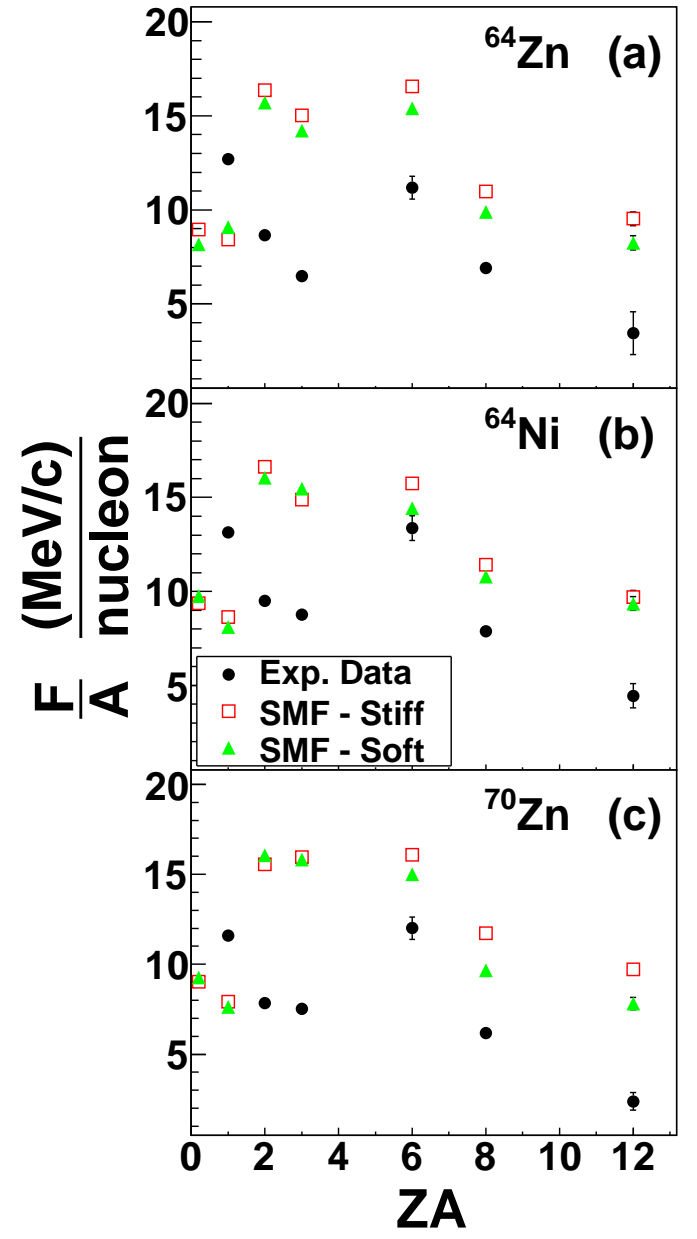

FIG. 7: (Color online) Flow parameter (F) per nucleon is shown as a function of the charge times mass (ZA) for the midperipheral collisions. The experimental data (black circles) is shown in comparison to the Stochastic Mean-Field model results, for both a stiff (red squares) and soft (green triangles) parameterization of the symmetry energy. The free neutron flow from the SMF model is offset with a $\mathrm{ZA}=0.1$ for clarity.

The largest discrepancy between the experimental and SMF results is that the proton flow in the SMF model is decreased relative to the ${ }^{2} \mathrm{H}$ and ${ }^{3} \mathrm{H}$ flows. This may be attributed to the overproduction of the free nucleons due to the lack of n-body correlations in the mean-field approach. Therefore, the additional free protons, that in reality should have been correlated with other nucleons, will decrease the flow relative to the correlated nucleons which are identified as fragments through the coalescence procedure.

The SMF model provides the opportunity to examine the sensitivity of the fragment flows to the density dependence of the symmetry energy. In Figure 7 the results from the SMF model are shown with both a stiff and soft $\mathrm{E}_{\text {sym }}(\rho)$. In general, the flow is decreased in the soft case, which can be attributed to the low density behavior of the symmetry potential. As discussed above, at low density the soft symmetry potential is more repulsive for neutrons and will, therefore, decrease the flow.

The difference between the ${ }^{3} \mathrm{H}$ and ${ }^{3} \mathrm{He}$ flow also appears to be sensitive to $\mathrm{E}_{\text {sym }}(\rho)$, as predicted previously by Scalone et al. [42]. In Figure 7 the stiff symmetry potential produces a larger ${ }^{3} \mathrm{He}$ flow than ${ }^{3} \mathrm{H}$ flow, while the opposite is shown with the soft symmetry potential for the ${ }^{64} \mathrm{Ni}$ and ${ }^{70} \mathrm{Zn}$ systems. In the ${ }^{64} \mathrm{Zn}$ system, the ${ }^{3} \mathrm{H}_{-}{ }^{3} \mathrm{He}$ difference is not very sensitive to $\mathrm{E}_{\text {sym }}(\rho)$ since the asymmetry of the system is lower and therefore the magnitude of the symmetry potential is smaller. It is also interesting to note that the ${ }^{3} \mathrm{H}_{-}{ }^{3} \mathrm{He}$ differences appear to be more sensitive to the density dependence of the symmetry energy than the free neutron-proton flows.

In order to quantitatively compare the ${ }^{3} \mathrm{H}-{ }^{3} \mathrm{He}$ flow between the experiment and SMF calculations the relative difference was calculated as,

$$
R_{3 H e-3 H}=\frac{F^{3 H e}-F^{3 H}}{F^{3 H e}+F^{3 H}}
$$

where $\mathrm{F}^{3 H e}$ and $\mathrm{F}^{3 H}$ represent the flow parameter extracted for the ${ }^{3} \mathrm{He}$ and ${ }^{3} \mathrm{H}$ fragments. Thus, if $\mathrm{R}_{3 \mathrm{He}-3 \mathrm{H}}$ is greater (less) than 0 than the ${ }^{3} \mathrm{He}$ flow is larger (smaller) than the ${ }^{3} \mathrm{H}$ flow. In Figure 8, $\mathrm{R}_{3 \mathrm{He}-3 \mathrm{H}}$ is shown from the experiment (blue fill area) and SMF model (circles) for the mid-peripheral collisions. The width of the blue fill area represents the fit error in extracting the transverse flow. The estimated systematic error in the experimental flow was accounted for and is shown by the dashed line. The largest uncertainty comes from the statistical/systematic error present in the ${ }^{3} \mathrm{He}$ flow. The SMF model results are shown both with (black circles) and without (gray circles) applying the detector thresholds. The results from the stiff and soft symmetry energy parameterizations are shown for each system as the closed and open circles, respectively.

The SMF model results, with and without the detector thresholds, demonstrate a sensitivity of the ${ }^{3} \mathrm{H}^{3} \mathrm{He}$ flow to the density dependence of the symmetry energy, specifically for the more neutron-rich ${ }^{64} \mathrm{Ni}$ and ${ }^{70} \mathrm{Zn}$ systems. Applying the detector thresholds to the simulated data produced an overall enhancement in the magnitude of $\mathrm{R}_{3 \mathrm{He}-3 \mathrm{H}}$, moving it in closer agreement with the experiment. In general, the ${ }^{3} \mathrm{H}-{ }^{3} \mathrm{He}$ difference is underestimated by the SMF model. However, a stiff density dependence of the symmetry energy provides the better agreement with the experimental data in all cases. While the SMF calculations do not reproduce the experimental data on absolute scale, the large $\mathrm{R}_{3 H e-3 H}$ values from the experiment favor the stiff form of $\mathrm{E}_{\text {sym }}(\rho)$. Thus, the results indicate that the LCP flow is likely a sensitive observable to the nuclear EoS. 


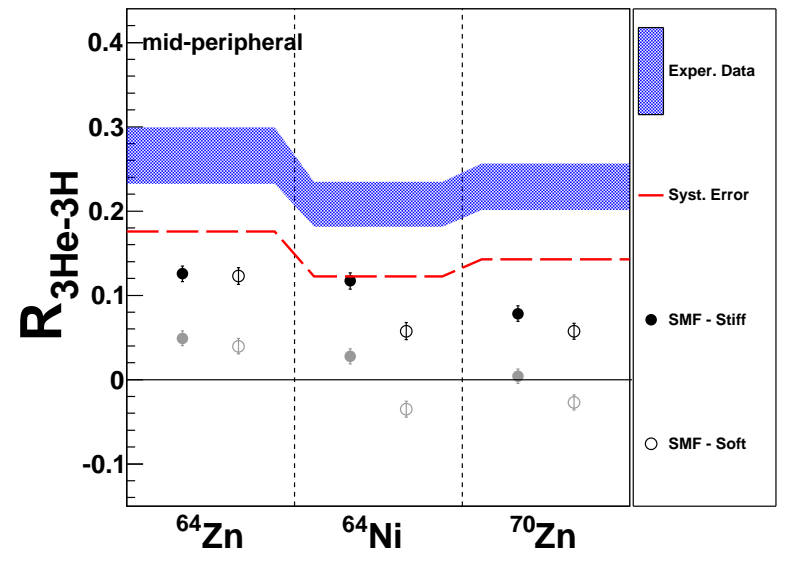

FIG. 8: (Color online) $\mathrm{R}_{3 H e-3 H}$, from Equation 3, is calculated from the ${ }^{64} \mathrm{Ni},{ }^{64} \mathrm{Zn}$, and ${ }^{70} \mathrm{Zn}$ systems for the midperipheral collisions. The results from the experimental data, including the fit error, are represented by the faded blue (gray) region. The estimated systematic error in the experimental results is represented by the red dashed line. The SMF results are shown with (black circles) and without (gray circles) applying the detector thresholds. As described by the legend, results with a soft and stiff density dependence of the symmetry energy are shown from the SMF model.

\section{Correlation between Mid-Rapidity Yield and Transverse Flow}

The mid-rapidity yield and transverse flow should be connected since both are observables associated with the emission of particles in between the QP and QT. For comparison, the two observables are shown, side-by-side, in Figure 6. A clear correlation is shown between the transverse flow (Figure 6a) and the inverse of $\mathrm{R}_{Y \text { ield }}^{\text {Mid }}$ (Figure $6 \mathrm{~b}$ ) of the LCPs. Nearly identical isotopic and isobaric trends are present. The results demonstrate that the increased relative mid-rapidity emission (a decrease in $\left.\left(R_{Y \text { ield }}^{M i d}\right)^{-1}\right)$ coincides with a decreased transverse flow. A possible explanation for the correlation based on the isospin migration phenomenon $[28,30]$ is presented below in context of the SMF model results.

The ${ }^{3} \mathrm{H}_{-}{ }^{3} \mathrm{He}$ flow results discussed above can be interpreted in terms of a more neutron-rich emission from the neck region in the stiff case [28]. The lower value of the stiff symmetry potential, with respect to the soft, during the expansion phase of the system leads to less repulsive dynamics, associated with a generally larger flow of the LCPs (see Figure 7). At the same time, the neutron excess is transferred towards the low-density neck region, from which light charged particles eventually emerge. The latter mechanism (isospin migration), that is sensitive to the derivative of the symmetry energy just below normal density, is more effective in the stiff case [28] producing a more neutron-rich neck region. This is confirmed by examining the $\mathrm{R}_{Y \text { ield }}^{\mathrm{Mid}}$ values of the
LCPs calculated from both the stiff and soft symmetry potential, as shown in Figure 3. An increase in the midrapidity yield of the neutron-rich fragments, ${ }^{3} \mathrm{H}$ and ${ }^{6} \mathrm{He}$, is observed in the stiff case, relative to the soft. The larger asymmetry of the neck region in the stiff case also explains the larger repulsion seen for ${ }^{3} \mathrm{H}$, with respect to ${ }^{3} \mathrm{He}$, thus producing a decreased flow (see Figures 7 and 8). This suggests a clear connection between the isobaric trends observed in the LCP flow and $\mathrm{R}_{Y \text { ield }}^{\mathrm{Mid}}$ results.

The importance of the neck dynamics, suggested by the SMF model, can also provide insight into the observation of the decreased flow with increasing neutron content of the LCPs (see Figs. 6-7). The isospin migration phenomenon represents the movement of neutrons and protons in opposite directions due to a density gradient. Thus, neutrons preferentially move towards the low-density neck region, while protons will travel towards a higher density region (near the saturation density), such as the QP. If the transverse flow is thought to represent the movement, or flow, of particles following the QP and QT, then the proton movement, due to the isospin migration, may enhance the transverse flow. Likewise, the opposite movement of neutrons could diminish the flow. Thus, upon coalescence of the free nucleons the proton-rich fragments, such as ${ }^{1} \mathrm{H}$ and ${ }^{3} \mathrm{He}$, would exhibit a larger flow with respect to neutron-rich fragments, such as ${ }^{3} \mathrm{H}$ and ${ }^{6} \mathrm{He}$. Therefore, the occurrence of isospin migration can account for the strong correlation observed between the LCP flow (Figure 6a) and mid-rapidity emission (Figure 6b). Further investigation of this concept is required, such as examining these observables in isospin asymmetric systems where isospin diffusion [77] is present and may enhance the observed trends.

\section{CONCLUSIONS}

In summary, the mid-rapidity emission and transverse flow of LCPs from $35 \mathrm{MeV} /$ nucleon $\mathrm{Zn}$ and $\mathrm{Ni}$ systems have been studied. Examination of the rapidity distributions of the LCPs demonstrated a strong preference for emission around the mid-rapidity region. Further examination showed an enhanced emission of the more neutron-rich isotopes and isobars in the mid-rapidity region, relative to the QP region, in comparison to the less neutron-rich LCPs. The transverse flow of the LCPs was also extracted and strong isotopic and isobaric trends, in which the flow decreased with increasing neutron content of the fragments. A strong correlation between the enhancement of the relative yield of the neutron-rich fragments in the mid-rapidity region and the decreased flow of the neutron-rich fragments was shown.

The SMF model was used to examine the mid-rapidity emission and transverse flow results, as well as the sensitivity of these observables to the density dependence of the symmetry energy. Many of the experimental trends were well reproduced by the SMF model, though, sig- 
nificant differences exist on absolute scale. The present studies demonstrate that both the mid-rapidity yield and transverse flow showed sensitivity to the density dependence of the symmetry energy. Additionally, the SMF results indicated that the correlation between the relative mid-rapidity yield and flow of the LCPs could be attributed to the occurrence of isospin migration.

\section{ACKNOWLEDGEMENTS}

We would like to thank the staff members of the Texas A\&M Cyclotron Institute for the excellent beam qual- ity. This work was supported in part by the Robert A. Welch Foundation through grant No. A-1266, and the Department of Energy through grant No. DE-FG0393ER40773. We would also like to thank the Target Lab at Argonne National Laboratory for the fabrication of the ${ }^{70} \mathrm{Zn}$ target and the Laboratory for Molecular Simulation at Texas A\&M University for providing computer time for the SMF calculations.
[1] S. Shlomo, V. M. Kolomietz, and G. Colo, Eur. Phys. J. A 30, 23 (2006).

[2] C. Fuchs and H. H. Wolter, Eur. Phys. J. A. 30, 5 (2006).

[3] B. A. Li, L. W. Chen, and C. M. Ko, Phys. Rep. 464, 113 (2008).

[4] M. B. Tsang, Y. Zhang, P. Danielewicz, M. Famiano, Z. Li, W. G. Lynch, and A. W. Steiner, Phys. Rev. Lett. 102, 122701 (2009).

[5] D. V. Shetty, S. J. Yennello, and G. A. Souliotis, Phys. Rev. C 76, 24606 (2007).

[6] S. Kowalski, J. B. Natowitz, S. Shlomo, R. Wada, K. Hagel, J. Wang, T. Materna, Z. Chen, Y. G. Ma, L. Qin, et al., Phys. Rev. C 75, 014601 (2007).

[7] J. M. Lattimer and M. Prakash, Science 304, 536 (2004).

[8] B. A. Li, Nucl. Phys. A708, 365 (2002).

[9] T. Klahn et al., Phys. Rev. C 74, 035802 (2006).

[10] R. J. Furnstahl, Nucl. Phys. A706, 85 (2002).

[11] J. R. Stone, J. C. Miller, R. Koncewicz, P. D. Stevenson, and M. R. Strayer, Phys. Rev. C 68, 034324 (2003).

[12] B. A. Li, Phys. Rev. Lett. 88, 192701 (2002).

[13] A. W. Steiner, M. Prakash, J. M. Lattimer, and P. J. Ellis, Phys. Rep. 411, 325 (2005).

[14] C. P. Montoya, W. G. Lynch, D. R. Bowman, G. F. Peaslee, N. Carlin, R. de Souza, C. K. Gelbke, W. B. Gong, et al., Phys. Rev. Lett. 73, 3070 (1994).

[15] R. Wada, M. Gonin, M. Gui, K. Hagel, Y. Lou, D. Utley, B. Xiao, D. Miller, J. B. Natowitz, et al., Nucl. Phys. A548, 471 (1992).

[16] J. Toke, B. Lott, S. P. Baldwin, B. M. Quednau, W. U. Schroder, L. G. Sobotka, J. Barreto, R. J. Charity, et al., Nucl. Phys. A583, 519 (1995).

[17] M. Di Toro, A. Olmi, and R. Roy, Eur. Phys. J. A 30 , 65 (2006).

[18] J. Lukasik, J. Benlliure, V. Metivier, E. Plagnol, B. Tarnian, M. Assenard, G. Auger, et al., Phys. Rev. C 55, 1906 (1997).

[19] E. Plagnol, J. Lukasik, G. Auger, C. O. Bacri, F. Bellaize, N.and Bocage, B. Borderie, et al., Phys. Rev. C 61, 014606 (1999).

[20] D. Theriault, J. Gautheir, F. Grenier, F. Moisan, C. St.Pierre, R. Roy, B. Davin, S. Hudan, et al., Phys. Rev. C. 74, 051602(R) (2006).

[21] J. F. Dempsey, R. J. Charity, L. G. Sobotka, G. J. Kunde, S. Gaff, C. K. Gelbke, T. Glasmacher, M. J. Huang, R. C. Lemmon, W. G. Lynch, et al., Phys. Rev. C 54, 1710
(1996).

[22] S. Piantelli, P. R. Maurenzig, A. Olmi, L. Bardelli, M. Bini, G. Casini, A. Mangiarotti, et al., Phys. Rev. C 76, 061601(R) (2007).

[23] R. Planeta, F. Amorini, A. Anzalone, L. Auditore, V. Baran, A. Benisz, I. Berceanu, A. Bonasera, et al., Phys. Rev. C. 77, 014610 (2008).

[24] S. Hudan, R. Alfaro, B. Davin, Y. Larochelle, H. Xu, L. Beaulieu, T. Lefort, R. Yanez, R. de Souza, R. J. Charity, et al., Phys. Rev. C 71, 054604 (2005).

[25] R. Laforest, E. Ramakrishnan, D. J. Rowland, A. Ruangma, E. M. Winchester, E. Martin, and S. J. Yennello, Phys. Rev. C 59, 2567 (1999).

[26] D. V. Shetty, A. Keksis, A. Ruangma, G. A. Souliotis, M. Veselsky, E. M. Winchester, S. J. Yennello, K. Hagel, Y. G. Ma, et al., Phys. Rev. C 68, 054605 (2003).

[27] J. Rizzo, M. Colonna, V. Baran, M. Di Toro, H. H. Wolter, and M. Zielinska-Pfabe, Nucl. Phys. A806, 79 (2008).

[28] R. Lionti, V. Baran, M. Colonna, and M. Di Toro, Phys. Lett. B 625, 33 (2005).

[29] V. Baran, M. Colonna, M. Di Toro, V. Greco, M. Zielinska-Pfabe, and H. H. Wolter, Nucl. Phys. A703, 603 (2002).

[30] V. Baran, M. Colonna, and M. Di Toro, Nucl. Phys. A730, 329 (2004).

[31] L. G. Sobotka, Phys. Rev. C 50, 1272(R) (1994).

[32] P. Danielewicz, R. Lacey, and W. G. Lynch, Science 298, 1592 (2002).

[33] D. J. Magestro, W. Bauer, and G. D. Westfall, Phys. Rev. C 62, 041603(R) (2000).

[34] B. A. Li, C. M. Ko, and W. Bauer, Int. J. Mod. Phys. E7, 147 (1998).

[35] M. Di Toro, S. J. Yennello, and B. A. Li, Eur. Phys. J. A 30, 153 (2006).

[36] V. Baran, M. Colonna, V. Greco, and M. Di Toro, Phys. Rep. 410, 335 (2005).

[37] Z. Kohley, L. W. May, S. Wuenschel, A. Bonasera, R. Hagel, K. Tripathi, R. Wada, G. A. Souliotis, D. V. Shetty, S. Galanopoulos, M. Mehlman, et al., Phys. Rev. C 82, 064601 (2010).

[38] R. Pak, W. Benenson, O. Bjarki, J. A. Brown, S. A. Hannuschke, R. A. Lacey, B. A. Li, et al., Phys. Rev. Lett. 78, 1022 (1997).

[39] R. Pak, B. A. Li, W. Benenson, O. Bjarki, J. A. Brown, 
S. Hannuschke, et al., Phys. Rev. Lett. 78, 1026 (1997).

[40] C. Liewen, Z. Fengshou, and J. Genming, Phys. Rev. C 58, 2283 (1998).

[41] B. A. Li, Z. Ren, C. M. Ko, and S. J. Yennello, Phys. Rev. Lett. 76, 4492 (1996).

[42] L. Scalone, M. Colonna, and M. Di Toro, Phys. Lett. B 461, 9 (1999).

[43] B. A. Li, Phys. Rev. Lett. 85, 4221 (2000).

[44] G. C. Yong, B. A. Li, and L. W. Chen, Phys. Rev. C 74, 064617 (2006).

[45] B. A. Li, Nucl. Phys. A708, 365 (2002).

[46] W. Trautmann, M. Chartier, Y. Leifels, R. C. Lemmon, Q. Li, J. Lukasik, A. Pagano, P. Pawlowski, P. Russotto, and P. Wu, Prog. Part. Nucl. Phys. 62, 425 (2009).

[47] W. Trautmann, M. Chartier, Y. Leifels, R. C. Lemmon, Q. Li, J. Lukasik, A. Pagano, P. Pawlowski, P. Russotto, and P. Z. Wu, arXiv:0907.2822v1 [nucl-ex] (2009).

[48] G. C. Yong, B. A. Li, L. W. Chen, and X. C. Zhang, Phys. Rev. C 80, 44608 (2009).

[49] J. Rizzo, M. Colonna, M. Di Toro, and V. Greco, Nucl. Phys. A732, 202 (2004).

[50] V. Giordano, M. Colonna, M. Di Toro, V. Greco, and J. Rizzo, Phys. Rev. C 81, 044611 (2010).

[51] S. Wuenschel, K. Hagel, R. Wada, J. Natowitz, S. J. Yennello, Z. Kohley, C. Bottosso, L. W. May, W. B. Smith, D. V. Shetty, et al., Nucl. Instrum. Methods Phys. Res. A 604, 578 (2009).

[52] R. P. Schmitt, L. Cooke, G. Derrig, D. Fabris, B. Hurst, J. B. Natowitz, G. Nebbia, D. OKelly, B. Srivastava, W. Turmel, et al., Nucl. Instrum. Methods Phys. Res. A 354, 487 (1995).

[53] J. Pouthas, B. Borderie, R. Dayras, E. Plagnol, M. F. Rivet, F. Saint-Laurent, J. C. Steckmeyer, G. Auger, C. O. Bacri, S. Barbey, et al., Nucl. Instrum. Methods Phys. Res. A 357, 418 (1995).

[54] K. Kwiatkowski, D. S. Bracken, K. B. Morley, J. Brzychczyk, E. Renshaw-Foxford, K. Komisarcik, V. E. Viola, N. R. Yoder, J. Dorsett, J. Poehlman, et al., Nucl. Instrum. Methods Phys. Res. A 360, 571 (1995).

[55] S. Wuenschel, K. Hagel, L. W. May, R. Wada, and S. J. Yennello, AIP Conf. Proc. 1099, 816 (2009).

[56] L. W. May, Z. Kohley, S. Wuenschel, K. Hagel, S. N. Soisson, G. A. Souliotis, B. C. Stein, R. Wada, and S. J. Yennello, To Be Submitted Nucl. Instrum. Methods Phys. Res. A (2010).
[57] L. Tassan-Got, Nucl. Instrum. Methods Phys. Res. B 194, 503 (2002).

[58] R. Wada, T. Keutgen, K. Hagel, Y. G. Ma, J. Wang, M. Murray, L. Qin, P. Smith, J. B. Natowitz, R. Alfarro, et al., Phys. Rev. C 69, 044610 (2004).

[59] S. Wuenschel, Ph.D. thesis, Texas A\&M University (2009).

[60] G. D. Westfall, Nucl. Phys. A630, 27 (1998).

[61] M. Papa, G. Giuliani, and A. Bonasera, J. Comput. Phys. 208, 403 (2005).

[62] W. K. Wilson, R. Lacey, C. A. Ogilvie, and G. D. Westfall, Phys. Rev. C 45, 738 (1992).

[63] P. Danielewicz and G. Odyniec, Phys. Lett. B 157, 146 (1985).

[64] D. Cussol, T. Lefort, J. Peter, G. Auger, C. O. Bacri, F. Bocage, B. Borderie, et al., Phys. Rev. C 65, 44604 (2002).

[65] C. A. Ogilvie, D. A. Cebra, J. Clayton, P. Danielewicz, S. Howden, J. Karn, et al., Phys. Rev. C 40, 2592 (1989).

[66] P. Chomaz, M. Colonna, and J. Randrup, Phys. Rep. 389, 263 (2004).

[67] E. Santini, T. Gaitanos, M. Colonna, and M. Di Toro, Nucl. Phys. A756, 468 (2005).

[68] A. Bonasera and L. P. Csernai, Phys. Rev. Lett. 59, 630 (1987).

[69] S. Gautam and A. D. Sood, Phys. Rev. C 82, 014604 (2010).

[70] G. D. Westfall, W. Bauer, D. Craig, M. Cronqvist, E. Gualtieri, S. Hannuschke, D. Klakow, et al., Phys. Rev. Lett. 71, 1986 (1993).

[71] A. Bonasera, F. Gulminelli, and J. Molitoris, Phys. Rep. 243, 1 (1994).

[72] R. Nebauer and J. Aichelin, Nucl. Phys. A650, 65 (1999).

[73] A. D. Sood and R. K. Puri, Phys. Rev. C 69, 054612 (2004).

[74] J. Zhang, S. Das Gupta, and C. Gale, Phys. Rev. C 50, 1617 (1994).

[75] A. Andronic, J. Lukasik, W. Reisdorf, and W. Trautmann, Eur. Phys. J. A 30, 31 (2006).

[76] J. Lukasik and W. Trautmann, arXiv:0603028 [nucl-ex] (2006).

[77] M. B. Tsang, T. X. Liu, L. Shi, P. Danielewicz, C. K. Gelbke, X. D. Liu, W. G. Lynch, W. P. Tan, G. Verde, A. Wagner, et al., Phys. Rev. Lett. 92, 062701 (2004). 